\title{
The Nature of Explosive Percolation Phase Transition
}

\author{
Liang Tian and Da-Ning Shi \\ College of Science, Nanjing University of Aeronautics and Astronautics, Nanjing 211106, China
}

(Dated: September 15, 2018)

\begin{abstract}
In this Letter, we show that the explosive percolation is a novel continuous phase transition. The order-parameter-distribution histogram at the percolation threshold is studied in Erdös-Rényi networks, scale-free networks, and square lattice. In finite system, two well-defined Gaussian-like peaks coexist, and the valley between the two peaks is suppressed with the system size increasing. This finite-size effect always appears in typical first-order phase transition. However, both of the two peaks shift to zero point in a power law manner, which indicates the explosive percolation is continuous in the thermodynamic limit. The nature of explosive percolation in all the three structures is belong to this novel continuous phase transition. Various scaling exponents concerning the order-parameter-distribution are obtained.
\end{abstract}

PACS numbers: 64.60.ah, 64.60.aq, 05.50.+q

Keywords: Explosive percolation; Phase transition; Finite-size effect; Order-parameter distribution

\section{INTRODUCTION}

Percolation [1], the simplest model presenting continuous phase transition, is one of the fundamental problems in statistical physics, since it provides deeper understanding of many other issues through Fortuin-Kasteleyn representation [2]. The percolation model itself has applications to a wide variety of different systems, ranging from sol-gel transition and polymerization [3, 4], to conductivity of composite materials and flow through porous media 5,6 , 6 , to spreading process and robustness in networks 7 -12]. Hitherto the critical properties in most of these systems are well described by the universality of percolation model in corresponding dimensionality.

Strikingly, Achlioptas, D'Souza, and Spencer [13] reported that the percolation transition for the ErdösRényi (ER) model [14] may become discontinuous, through a modified growth procedure known as product rule (PR). They found at the percolation threshold an abrupt jump in the size of the largest component, which was named as explosive percolation (EP) compared with the traditional continuous percolation transition. In light of this, subsequent studies were devoted to uncovering the underlying mechanism of EP [15], proposing new models for EP [16 21], and studying EP transition with different topologies and dimensionalities [22 26]. Recently, two empirical studies focused on the EP in human protein network [27] and social network [28].

While further investigations confirmed the abrupt transition in EP, it was also shown that the critical distribution of cluster sizes follows a power law [26], which manifests the features characteristic of the second-order phase transition. The contradictions make the nature of explosive percolation transition a controversial issue in statistical physics, which needs to be clarified. Recent research proves that the explosive percolation is a weak continuous phase transition in mean-field structure 29 32]. It is reminiscent of the weak first-order phase transition [33] in five-state Potts model [34], where, since the correlation length is very large at transition point, the accessible system size in numerical simulation is always in the critical region, and thus the picture of cluster distribution is characterized by fractal shapes rather than smooth droplets. The weak first-order phase transition is hard to establish due to its proximity and resemblance to a critical point, and weak continuous phase transition is hard to confirmed due to the smallness of the critical exponent for the order parameter.

However, in this Letter, we show that the explosive percolation is not a traditional weak continuous phase transition by examining the distribution histogram of the order parameter $G$ defined as the fraction of vertices in the largest cluster. Three structures are considered, such as ER network, scale-free (SF) network, and two-dimensional (2D) lattice. Three key features are observed in all these structures. Firstly, we find that in finite system two well-defined Gaussian-like peaks coexist in the order-parameter-distribution histogram at the percolation threshold, which represent the nonpercolative phase and percolative phase, respectively. Secondly, the probability of realizing a configuration in the intermediate phase between the two peaks is suppressed as a power law with the system size increasing. Finally, both of the two peaks shift to zero point in power law manner. These observations indicate the explosive percolation is a continuous phase transition with first-order-like finite-size effect. Various scaling exponents concerning order-parameter-distribution are obtained.

\section{METHOD}

For concreteness, numerical simulations were performed according to the original PR process [13]: In each turn, two unoccupied edges are randomly chosen; the one which minimizes the product of the masses of the clusters it joins is retained. For square lattice, we imposed periodic boundary conditions in both directions to reduce the boundary effect. For SF network, we adopted the model by Chung and Lu (CL) 36 to build the network. Specif- 

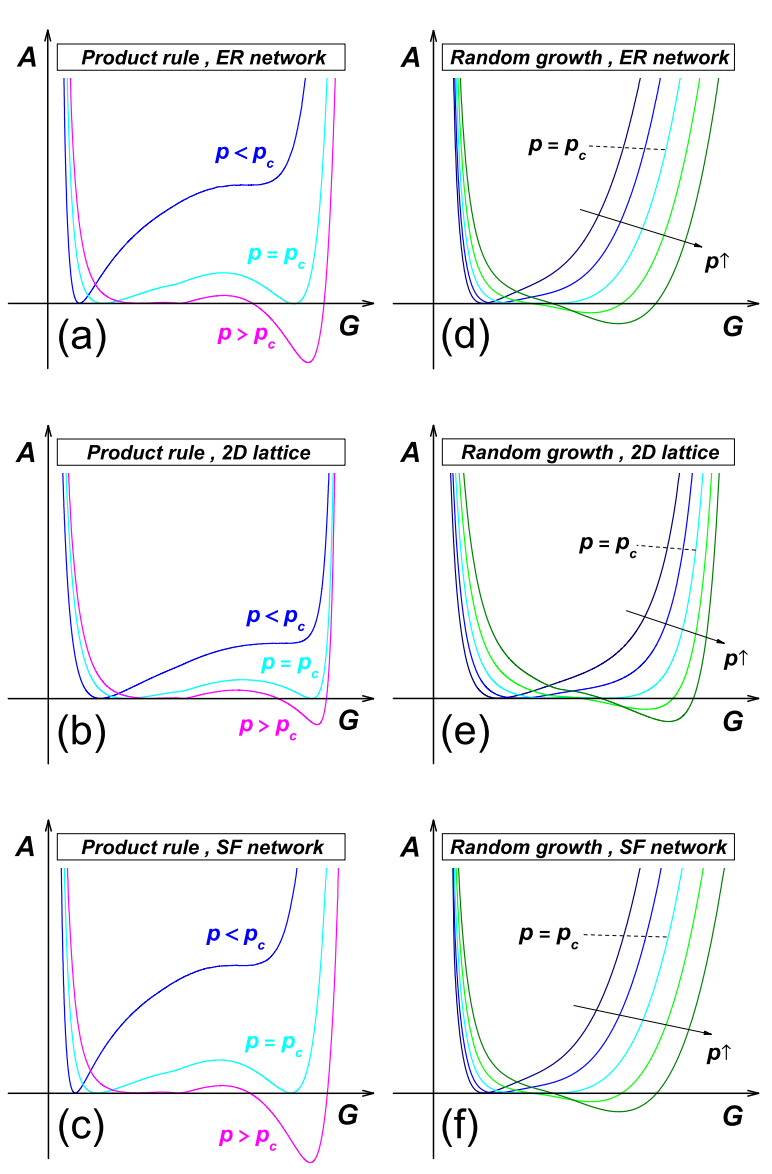

FIG. 1. Plots of $A(G, p)$ as a function of order parameter $G$ in the vicinity of percolation threshold $p_{c}$ for PR model (a) (b) (c) and traditional RG model (d) (e) (f). The exponent $\lambda=2.8$ is used for SF network. The system size is 4096 for all the simulations in this figure. The full information of $p$ can be found in the movies of order parameter distribution in the vicinity of transition point [35].

ically, every vertex in the system is assigned a weight beforehand according to the desired degree distribution, and at every time step, two edges are independently selected with probability proportional to the product of the weights of the vertices at the end of each edge. Then the $\mathrm{PR}$ is used to decide which is the next occupied edge.

The controlling parameter $p$ denotes the number of added edges divided by the system size $N$. We measured the order-parameter-distribution histogram $H(G, p)$ for each $p$ through extensive Monte Carlo (MC) simulations. According to the standard probability theory, the number of realized configurations with order parameter $G$ is

$$
H(G, p)=\exp [-A(G, p)] \sim Z^{-1}(p) Q(G, p),
$$

where $Z(p)$ is the normalization factor and $Q(G, p)$ is the order-parameter probability density function, i.e., the probability that, after $p N$ edges are added with PR process, the fraction of vertices in the largest cluster is $G$. When the number of realizations increases to infinity,
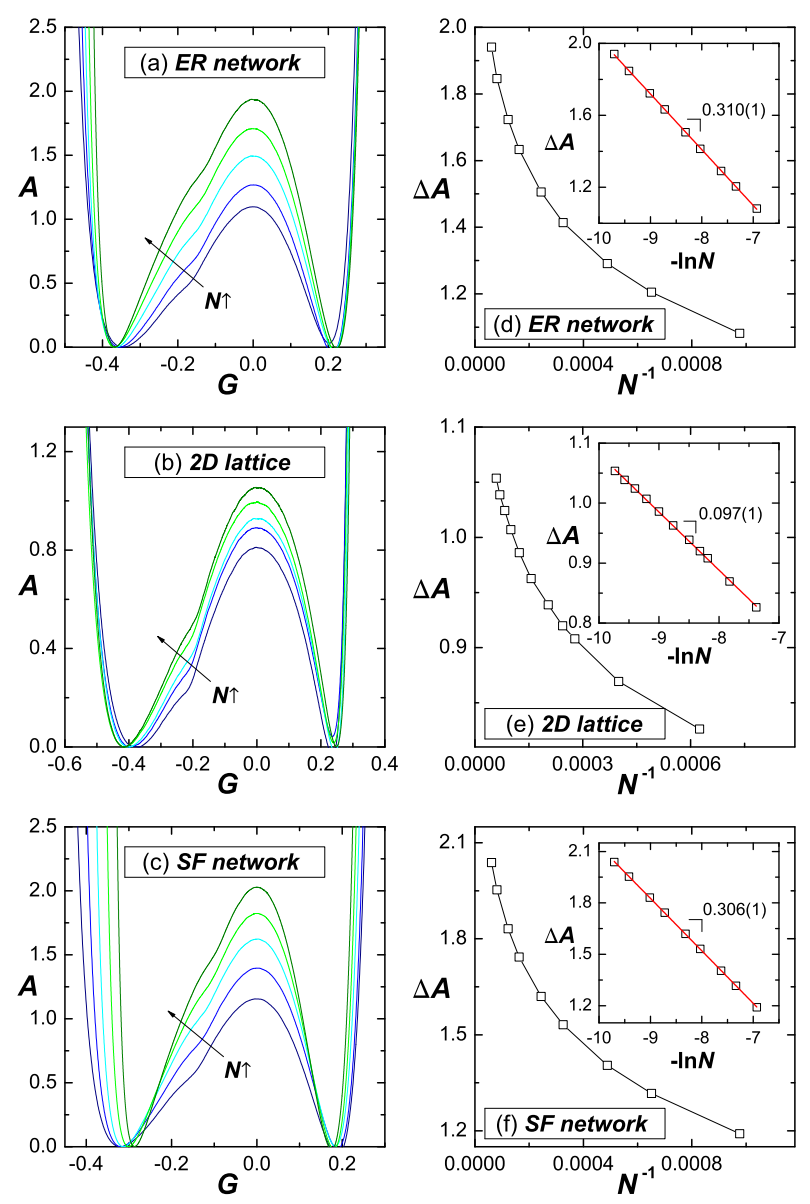

FIG. 2. The dependence of $A(G, p)$ at percolation threshold on the order parameter $G$ for different system sizes (left panels). The values of the minima have been normalized to 0 , and the curves have been translated along horizontal axis for better comparisons. The system sizes $N=$ 1024, 2048, 4096, 8192, 16384 for (a) ER network and (c) SF network, and $N=1600,2500,4900,8100,16900$ for (b) 2D lattice. Right panels display the depth of the minima in $A\left(G, p_{c}\right)$ as a function of system size. The red lines in the insets are the linear fits. The exponent $\lambda=2.8$ is used for SF network.

$H(G, p)$ is identical to $Q(G, p)$ multiplied by a constant. Intuitively, we have $A(G, p)=-\ln H(G, p)$, and thus at a given $p$ the location of the global minimum in $A(G, p)$ denotes the most probable size of the giant component.

\section{RESULT AND DISCUSSION}

Our computer implementation makes use of the effective Newman-Ziff algorithm [37] for tracking the largest cluster in the system. We carried out $10^{6} \mathrm{MC}$ sweeps per vertex to achieve high statistical accuracy for $H(G, p)$. In Fig. 1, we show the behavior of $A(G, p)$ near the percolation threshold $p_{c}$ for both PR model and traditional random growth (RG) model. It is well known that the percolation transition with RG is continuous, which is 


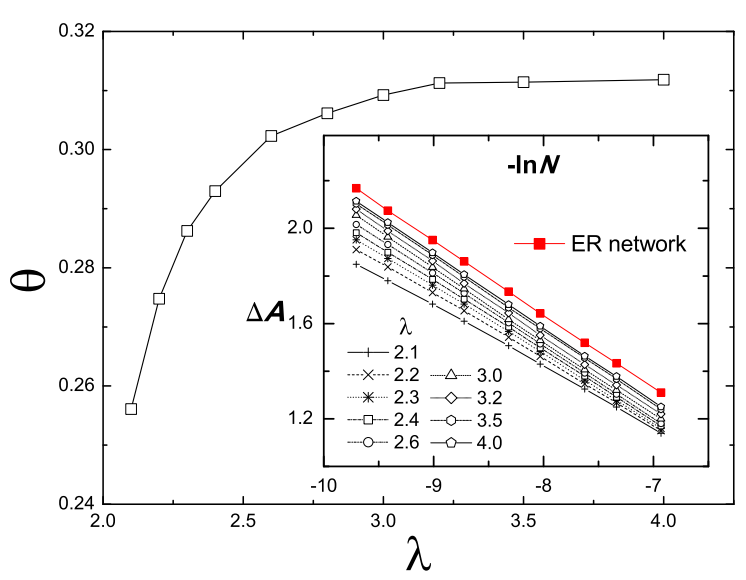

FIG. 3. The inset shows in SF network the size-dependent depth of minima in $A(G, p)$ at percolation threshold for different $\lambda$. The red solid squares represent the same relation in ER network. The fitting exponent $\theta$ as a function of $\lambda$ is displayed in the main panel.

reproduced in the simulations (see Fig. 1 (d) (e) (f)). As $p$ passes through the percolation threshold, there is only one global minimum in $A(G, p)$, which implies the order parameter grows continuously from one phase to the other. For PR model, the situation is completely different. As $p$ goes below the critical value, a local minimum appears in the region of large order parameter, and its value gradually approaches that of the global one. Right at the percolation threshold $p_{c}$, the two minima have equal depth, indicating that the nonpercolative and percolative configurations are realized with equal probability. When $p$ is beyond $p_{c}$, the second minimum becomes global and percolative phase dominates. The physical picture of the whole process [35] is reminiscent of the Landau theory of first-order phase transition. This is the first finite-size property of explosive percolation.

In the following, we perform detailed finite-size scaling analysis of $A\left(G, p_{c}\right)$. An important quantity is the depth of the minima (corresponding to the peaks in $H(G, P)$ ), $\Delta A$, relative to the local maximum (corresponding to the valley in $H(G, P)$ ) in-between corresponding to the intermediate phase. For typical first-order phase transitions [38, 39], with the system size increasing, $\Delta A$ also monotonically increases as the minima gradually develop, and eventually goes to infinity in the thermodynamic limit. We show here that this property also exists in explosive percolation. Since $p$ denotes the number of added edges which is not continuous, it is more convenient to calculate the depth with $\Delta A=A_{\max }-\left(A_{\min }^{1}+A_{\min }^{2}\right) / 2$, where $A_{\max }$ is the value of the local maximum and $A_{\min }^{1}$ and $A_{\min }^{2}$ are those of the two minima. For the determination of $A_{\min }^{1}$ and $A_{\min }^{2}$, it should be sufficient to use quadratic fit in the vicinity of the minima corresponding to the gaussian-like peaks in $H\left(G, p_{c}\right)$. However, $A\left(G, p_{c}\right)$ shows sizable asymmetry near the minima, thus we use cubic fit to obtain more accurate results. To determine
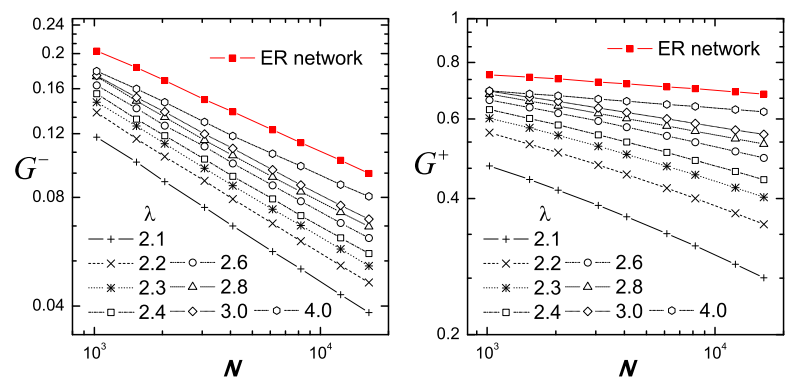

FIG. 4. The locations of the two minima $G^{-}$(left) and $G^{+}$ (right) as functions of system size $N$ for scale-free networks and ER network.

the value of $A_{\max }$, we found it adequate to use the same fit. Figure 2 shows the simulation results for the sizedependent behavior of $A\left(G, p_{c}\right)$. Indeed, the depth of the minima monotonically increases with system size, and tends towards infinity in the thermodynamic limit. Furthermore, a clear relation between $\Delta A$ and the logarithm of $N$ is observed,

$$
\Delta A \sim \theta \ln N
$$

In other words, the relative probability of finding a configuration in the intermediate phase is suppressed, as the system size increases, in power-law manner with exponent $\theta$. It should be noticed that $\theta_{E R}=0.310(1)$ for ER network and $\theta_{2 D}=0.097(1)$ for square lattice are very different. For SF network, this scaling relation holds for $\lambda>2.0$ (see the inset of Fig. 3). As $\lambda$ increases, the value of exponent $\theta$ gradually approaches that for ER network, and at $\lambda \rightarrow \infty$ the CL model is identical to ER model. In fact, $\theta(\lambda)$ is already saturated for $\lambda>3.0$ (see Fig. 3), where with PR the SF network generated by CL model is hardly distinguishable from ER network [24, 26]. The exponent $\theta$ for different structures is listed in Table प. This is the second finite-size property of explosive percolation.

The locations of the two minima in order-parameter dimension, $G^{+}$and $G^{-}$, directly measures the jump of the order parameter at percolation threshold. In all the three structures, both of $G^{+}$and $G^{-}$shift to zero in a power law manner, $G^{ \pm} \sim \eta^{ \pm}$. As $N$ increases, the two minima gradually get close to each other, and in the thermodynamic limit they merge at the transition point of the order parameter, where it presents a continuous phase transition. Actually, in the research of explosive percolation on scale-free network where another field, the degree distribution exponent $\lambda$, comes into play, it was claimed that there exists a tricritical point (TP) at $\lambda_{c} \in(2.3,2.4)$, above which the EP transition is first order [24]. At the same time, however, careful finite-size scaling analysis implied that for $\lambda<3.0$ the EP transition is continuous [25]. Our results indicate that explosive percolation in scale-free network is continuous in the whole range of $\lambda$. The scaling behavior of $G^{ \pm}$in scale-free networks

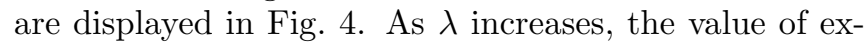
ponents $\eta^{ \pm}$gradually approaches those for ER network. 
TABLE I. Summary of scaling exponents obtained from our numerical analysis.

\begin{tabular}{lccc}
\hline \hline System & $\eta^{-}$ & $\eta^{+}$ & $\theta$ \\
\hline SF $\lambda=2.1$ & $0.404(1)$ & $0.205(3)$ & $0.256(2)$ \\
SF $\lambda=2.2$ & $0.389(2)$ & $0.168(2)$ & $0.274(1)$ \\
SF $\lambda=2.3$ & $0.376(2)$ & $0.145(1)$ & $0.286(1)$ \\
SF $\lambda=2.4$ & $0.366(2)$ & $0.129(1)$ & $0.292(1)$ \\
SF $\lambda=2.6$ & $0.351(2)$ & $0.106(1)$ & $0.302(1)$ \\
SF $\lambda=2.8$ & $0.343(2)$ & $0.091(1)$ & $0.306(1)$ \\
SF $\lambda=3.0$ & $0.331(2)$ & $0.079(1)$ & $0.309(1)$ \\
SF $\lambda=3.2$ & $0.293(1)$ & $0.047(1)$ & $0.311(1)$ \\
SF $\lambda=3.5$ & $0.291(2)$ & $0.041(1)$ & $0.311(1)$ \\
SF $\lambda=4.0$ & $0.290(2)$ & $0.038(1)$ & $0.311(1)$ \\
ER network & $0.282(1)$ & $0.035(1)$ & $0.310(1)$ \\
2D lattice & $0.085(3)$ & $0.013(1)$ & $0.097(1)$ \\
\hline \hline
\end{tabular}

The exponents $\eta^{ \pm}$for different structures are listed in Table [1]

\section{CONCLUSION}

In this Letter, we show that the explosive percolation is a novel continuous phase transition in ER network, scale- free network, and 2D lattice. By examining the orderparameter-distribution histogram at percolation threshold, it is found that two well-defined Gaussian-like peaks coexist, which represent the nonpercolative phase and percolative phase, respectively. Moreover, the probability of realizing a configuration in the intermediate phase between the two peaks is suppressed as a power law with the system size increasing. On the other hand, two peaks gradually get close to each other with the system size increasing, and in the thermodynamic limit they merge at the transition point of the order parameter. These observations suggest the explosive percolation is a continuous phase transition with first-order-like finite-size effect.

\section{ACKNOWLEDGMENTS}

The author (L. T.) thanks Robert Ziff, Peter Grassberger, Bernardo Huberman, and Lei-Han Tang for stimulating discussions and suggestions. The financial supports from CX07B-033z and BCXJ07-11 are acknowledged.
[1] D. Stauffer and A. Aharony, Introduction to Percolation Theory, 2nd ed. (Taylor \& Francis, London, 1994).

[2] C. M. Fortuin and P. Kasteleyn, Physica (Amsterdam) 57, 536 (1972).

[3] C. J. Brinker and G. W. Scherer, Sol-Gel Science: The Physics and Chemistry of Sol-Gel Processing (Academic, New York, 1990).

[4] R. M. Ziff, E. M. Hendriks, and M. H. Ernst, Phys. Rev. Lett. 49, 593 (1982).

[5] M. Sahimi, Applications of Percolation Theory, 2nd ed. (Taylor \& Francis, London, 1994).

[6] J. S. Andrade, S. V. Buldyrev, N. V. Dokholyan, S. Havlin, P. R. King, Y. K. Lee, G. Paul, and H. E. Stanley, Phys. Rev. E 62, 8270 (2000).

[7] C. Moore and M. E. J. Newman, Phys. Rev. E 61, 5678 (2000).

[8] R. Pastor-Satorras and A. Vespignani, Phys. Rev. Lett. 86, 3200 (2001).

[9] J. Shrager, T. Hogg, and B. A. Huberman, Science 236, 1092 (1987).

[10] R. Albert, H. Jeong, and A.-L. Barabási, Nature (London) 406, 378 (2000).

[11] R. Cohen, K. Erez, D. ben Avraham, and S. Havlin, Phys. Rev. Lett. 85, 4626 (2000).

[12] A. A. Moreira, J. S. Andrade, H. J. Herrmann, and J. O. Indekeu, Phys. Rev. Lett. 102, 018701 (2009).

[13] D. Achlioptas, R. M. D'Souza, and J. Spencer, Science 323, 1453 (2009).

[14] P. Erdős and A. Rényi, Publ. Math. Hugar. Acad. Sci. 5, 7 (1960).

[15] E. J. Friedman and A. S. Landsberg, Phys. Rev. Lett. 103, 255701 (2009).
[16] A. A. Moreira, E. A. Oliveira, S. D. S. Reis, H. J. Herrmann, and J. J. S. Andrade, Phys. Rev. E 81, 040101(R) (2010).

[17] R. M. D’Souza and M. Mitzenmacher, Phys. Rev. Lett. 104, 195702 (2010).

[18] N. A. M. Araújo and H. J. Herrmann, Phys. Rev. Lett. 105, 035701 (2010).

[19] Y. S. Cho, B. Kahng, and D. Kim, Phys. Rev. E 81, 030103(R) (2010).

[20] Y. S. Cho, S.-W. Kim, J. D. Noh, B. Kahng, and D. Kim, Phys. Rev. E 82, 042102 (2010).

[21] S. S. Manna and A. Chatterjee, Physica A 390, 177 (2011).

[22] R. M. Ziff, Phys. Rev. Lett. 103, 045701 (2009).

[23] R. M. Ziff, Phys. Rev. E 82, 051105 (2010).

[24] Y. S. Cho, J. S. Kim, J. Park, B. Kahng, and D. Kim, Phys. Rev. Lett. 103, 135702 (2009).

[25] F. Radicchi and S. Fortunato, Phys. Rev. Lett. 103, 168701 (2009).

[26] F. Radicchi and S. Fortunato, Phys. Rev. E 81, 036110 (2010).

[27] H. D. Rozenfeld, L. K. Gallos, and H. A. Makse, Eur. Phys. J. B 75, 305 (2010).

[28] R. K. Pan, M. Kivela, J. Saramaki, K. Kaski, and J. Kertész, Phys. Rev. E 83, 046112 (2011).

[29] R. A. da Costa, S. N. Dorogovtsev, A. V. Goltsev, and J. F. F. Mendes, Phys. Rev. Lett. 105, 255701 (2010).

[30] P. Grassberger, C. Christensen, G. Bizhani, S.-W. Son, and M. Paczuski, Phys. Rev. Lett. 106, 225701 (2011).

[31] O. Riordan and L. Warnke, Science 333, 322 (2011).

[32] H. K. Lee, B. J. Kim, and H. Park, Phys. Rev. E 84, 020101 (2011). 
[33] B. Nienhuis, A. N. Berker, E. K. Riedel, and M. Schick, Phys. Rev. Lett. 43, 737 (1979).

[34] F. Y. Wu, Rev. Mod. Phys. 54, 235 (1982).

[35] See the supporting material for the movies of $H(G, p)$ in the vicinity of percolation threshold for PR model.

[36] F. Chung and L. Lu, Annals of Combinatorics 6, 125 (2002).
[37] M. E. J. Newman and R. M. Ziff, Phys. Rev. Lett. 85, 4104 (2000).

[38] K. Binder, Rep. Prog. Phys. 50, 783 (1987).

[39] J. Lee and J. M. Kosterlitz, Phys. Rev. Lett. 65, 137 (1990). 